\title{
Zinner syndrome: a rare embryological anomaly of the mesonephric ducts - Report of two cases and literature review
}

\author{
Miguel A. Reyes-Caldelas* and Mauricio A. García-De León \\ Department of Radiology and Imaging, Hospital General de México, Mexico City, Mexico
}

\begin{abstract}
We present the cases of two males in their forties who present with symptoms or previous history of urolithiasis and known unilateral renal agenesis. On computed tomography evaluations of both patients, cystic lesions of the seminal vesicles were seen with further evaluation by transrectal ultrasound, making the diagnosis of Zinner syndrome. This entity has an embryological background that cannot be unseen. The symptoms are very characteristic, but incidental diagnosis based on imaging is very frequent. Good outcomes by laparoscopic treatment have been described.
\end{abstract}

Key words: Zinner syndrome. Renal agenesis. Seminal vesicle cyst.

\section{Introduction}

Zinner syndrome is a rare congenital anomaly usually presented in young men with a variety of symptoms. The image findings are very characteristic and new treatments have been documented in the past few years.

\section{Presentation of clinical cases}

\section{Case 1}

A 36-year-old male, with a history of known left renal agenesis, underwent a computed tomography (CT) scan on February 2017 for the evaluation of the right renal lithiasis and status of a double-J ureteral catheter seen on plain abdomen X-ray. The CT demonstrated agenesis of the left kidney and increase in volume of seminal vesicles with the left predominance, so it was integrated the diagnosis of Zinner syndrome, transrectal ultrasound was performed where a cystic aspect of the left seminal vesicle is observed (Fig. 1). Two months later, extracorporeal lithotripsy was performed with a torpid response and evolution with self-referral of the patient to private practice hospital on November 2017. No fertility problems were referred by the patient.

\section{Case 2}

A 37-year-old male was initially evaluated in December 2016 for presenting with a history of right renal fossa pain of 4 months of duration, suggestive of renal lithiasis; a renal ultrasound was performed where a stone in the distal ureter was observed. Treatment was started by placing a double right $\mathrm{J}$ catheter, which is observed in plain abdominal radiography adjacent to the lithium, for posterior realization of the right ureteroscopy; however, the patient suffers again from pain in the right renal fossa, fever, and non-clot forming hematuria, so a new contrast-enhanced CT scan was performed seeing right ureteral lithiasis, left seminal vesicle cyst and homolateral renal agenesis, concluding Zinner syndrome, transrectal ultrasound is performed in which the

\section{Correspondence:}

*Miguel A. Reyes-Caldelas

E-mail: reyescaldelasmiguelangel@gmail.com NC-ND license (http://creativecommons.org/licenses/by-nc-nd/4.0/).

Date of reception: 15-01-2019

Date of acceptance: 30-10-2019 DOI: 10.24875/HGMX.19000018
Available online: 17-01-2020 Rev Med Hosp Gen Mex. 2020;83(1):38-41 www.hospitalgeneral.mx 
seminal vesicles are best characterized (Fig. 2). The fever yields to antibiotic therapy and is discharged for follow-up by the outpatient clinic, where resection of the left seminal vesicle cyst was done laparoscopically, being discharged after 3 days, on June 2017. The reason of the surgery was no clearly specified. No fertility problems were referred by the patient.

\section{Discussion}

Seminal cyst was described initially by Smith in 1872 and then the association with ipsilateral renal agenesis described by Zinner in 1914. By 2009, 200 cases have been reported in literature, from that date to 2018, we counted on PubMed 31 cases, without counting these two, giving an approximate of 233 cases in total. In 2009 in Taipei, Seo et al. reported an incidence of $0.0046 \%$. Furthermore, there were reported about 50 cases in pediatric age in literature up to now, only $12 / 50$ were diagnosed in the $1^{\text {st }}$ year, and more than $80 \%$ were asymptomatic at long-term follow-up. It is a rare entity usually discovered when imaging evaluation is performed for other purposes, but sometimes is presented with symptomatology, and is man in the third or fourth decade of life the most affected, maybe because they are at the period of greatest reproductive or sexual activity, being found $83 \%$ of the cases between 11 and 40 years. We found it very often associated with infertility, painful ejaculation/dysuria or hemospermia, cryptorquidism, obstructed vas deferens mimicking paratesticular mass, and neoplasms in the urinary tract including in this last one malignant degeneration of the cyst ${ }^{1-25}$.

This syndrome is explained by the common embryologic origin of both organs (kidneys and seminal vesicles) from the mesonephric duct and is due to an insult in embryogenesis between the $4^{\text {th }}$ and $13^{\text {th }}$ weeks of gestation. In the female, it is presented as an obstructed hemivagina and ipsilateral renal anomaly, also called OHVIRA syndrome. The ureteral bud arises from the dorsal part of the distal mesonephric duct and extends dorsocranially to meet and induce differentiation of the metanephric blastema, from which the kidney will develop. The mesonephric duct will differentiate to epididymis, ejaculatory duct, vas deferens, seminal vesicle, and hemitrigone. Complete failure of the mesonephric duct results to the absence of the kidney, ureter hemitrigone, and seminal vesicle. Anomalous development of the distal mesonephric duct results in atresia of the ejaculatory ducts and abnormal ureteral budding; the former leads to obstruction and cystic dilatation of the seminal vesicles with the development of seminal

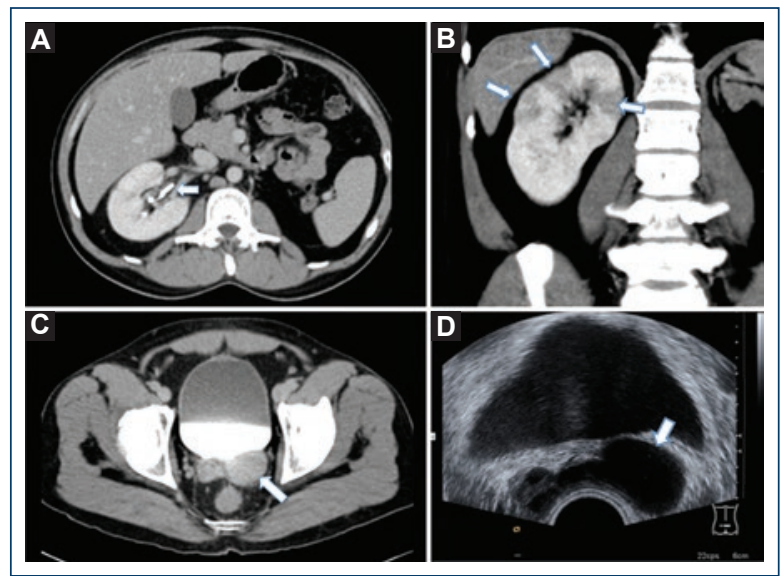

Figure 1. A: we can appreciate an axial image of a contrast-enhanced computed tomography where the absence of the left kidney is seeing and a double-J ureteral stent is noted on the right kidney (white arrow). B: a coronal image of a contrast-enhanced phase demonstrates wedge-shaped areas that are hypodense in comparison to the rest of the parenchyma (white arrows), consistent with an inflammatory process. C: we see an axial image of the same study at the level of the pelvis on excretory phase (note the contrast on the bladder) and the presence of dilated seminal vesicles of the left predominance (white arrow). D: a transrectal ultrasound on axial plane is displayed demonstrating the same anatomic level on C, with an enlarged seminal vesicle of cystic appearance (white arrow).

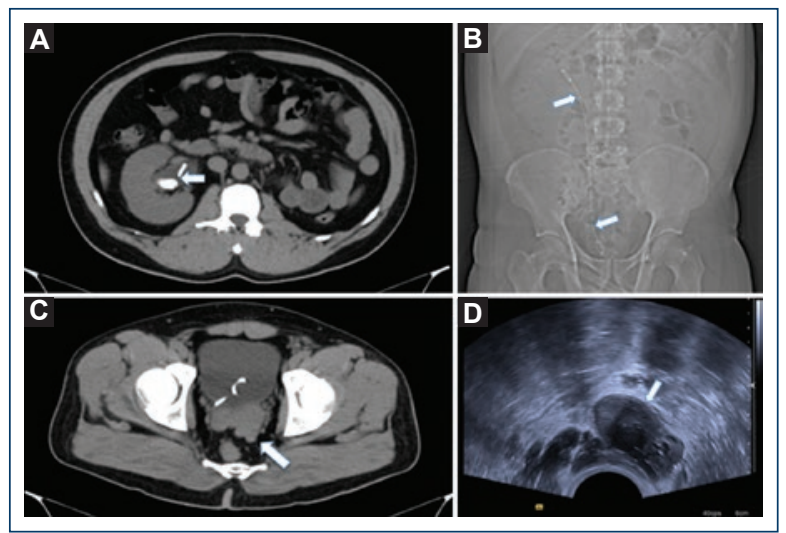

Figure 2. A: we can appreciate an axial image of a non-enhanced computed tomography where the absence of the left kidney is seeing and stone near a double-J ureteral stent (white arrow) on an enlarged kidney. B: the whole double-J ureteral stent (white arrows). C: we see an axial image of the same study at the level of the pelvis, with the tip of the ureteral stent on the bladder and presence of dilated seminal vesicles of the left predominance (white arrow). D: a transrectal ultrasound on axial plane is displayed demonstrating the same anatomic level on C, with an enlarged seminal vesicle of cystic appearance (white arrow), where low-level echoes are seeing. 
cyst, the latter leads to renal agenesis or dysplasia. Unilateral renal agenesis is present in $0.1 \%$ of newborns and should alert for other genitourinary anomalies present in up to $30-40 \%$ of these patients. The ureter can be absent, incomplete, or may have an ectopic course to the seminal vesicle (present in $36 \%$ of the cases) $)^{3,21-23}$.

Cyst smaller than $5 \mathrm{~cm}$ is usually asymptomatic and is discovered incidentally. Larger cysts can produce a wide variety of urological symptoms; usually, they measure more than $12 \mathrm{~cm}$ and can result in bladder or colon obstruction. The most affected side has been reported to be the right with a right: left ratio of 2:1. In both of our patients, the image findings were merely casual due to prostate hyperplasia treatment protocol evaluation through endorectal ultrasound and the other one due to known renal agenesis under CT evaluation. Findings by transrectal ultrasound include anechoic pelvic mass with a thick, irregular wall and occasional wall calcifications, or the mass may contain internal debris reflecting prior hemorrhage or infection. Magnetic resonance imaging (MRI) is paramount in making the definitive diagnosis, appearing as T2-weighted signal hyperintensity structures and T1-weighted signal hypointensity structures ${ }^{3,21}$.

Differential diagnosis includes Mullerian cyst, ureterocele, dilated ureter, abscess or lymph nodes, and acquired seminal vesicle cysts. The last ones are often bilateral and related to older patients that have undergone prostate surgery or with chronic prostatitis ${ }^{3,21}$.

Treatment is considered only for symptomatic patients and is surgical. Differential options exist from less invasive transrectal or transperineal aspiration of the cyst which gives a transitory relief of symptoms. The better outcome is reached by vesiculectomy with resection of ureteral or renal remnants if present and is well-described laparoscopically with low morbidity and good results in terms of symptoms relief and semen parameters; it has been even described using robot-assisted excision with the da Vinci robotic surgical system. No improvement in renal function has been observed, but a significant change in fertility rates has been documented on several cases in the mid-term 2,3,7-10,15.

\section{Conclusion}

This is a syndrome that must be suspected on imaging when renal agenesis and ipsilateral seminal cyst are present. A complete evaluation by ultrasound can be easily done and on CT or MRI extent of the region can be done for correct assessment. The spectrum of symptoms is well described and obligates the clinician for imaging evaluation. Resection by laparoscopic approach is of choice to symptomatic patients.

\section{Conflicts of interest}

The authors declare that they have no conflicts of interest.

\section{Ethical disclosures}

Protection of human and animal subjects. The authors declare that no experiments were performed on humans or animals for this study.

Confidentiality of data. The authors declare that they have followed the protocols of their work center on the publication of patient data.

Right to privacy and informed consent. The authors have obtained the written informed consent of the patients or subjects mentioned in the article. The corresponding author is in possession of this document.

\section{References}

1. Ahallal Y, Tazi MF, Khallouk A, Elammari J, Elfassi MJ, Farih MH. Conservative management of a congenital seminal vesicle cyst associated with ipsilateral renal agenesis revealed by cystitis: one case report. Case Rep Urol. 2011;2011:125753.

2. Naval-Baudin P, Carreño García E, Sanchez Marquez A, Valcárcel José J, Romero NM. Multicystic seminal vesicle with ipsilateral renal agenesis: two cases of Zinner syndrome. Scand J Urol. 2017;51:81-4.

3. Pereira BJ, Sousa L, Azinhais $P$, Conceição $P$, Borges R, Leão R, et al. Zinner's syndrome: an up-to-date review of the literature based on a clinical case. Andrologia. 2009;41:322-30.

4. Seo IY, Kim HS, Rim JS. Congenital seminal vesicle cyst associated with ipsilateral renal agenesis. Yonsei Med J. 2009;50:560-3.

5. Sundar R, Sundar G. Zinner syndrome: an uncommon cause of painful ejaculation. BMJ Case Rep. 2015;2015:1-5.

6. Haddock P, Wagner JR. Seminal vesicle cyst with ipsilateral renal agenesis and ectopic ureter (Zinner syndrome). Urology. 2015;85:e41-2.

7. Andrade-Rocha FT. Semen analysis in an infertile man with seminal vesicles cysts associated with ipsilateral renal agenesis. Int Urol Nephrol. 2006;38:101-3.

8. Khanduri S, Katyal G, Sharma H, Goyal A, Singh N, Yadav H. Unique association of multiple seminal vesicle cysts with contralateral renal agenesis: a rare variant of Zinner syndrome. Cureus. 2017;9:e1415.

9. Aghaways I, Ahmed SM. Endourologic intervention for management of infertility in a man with Zinner syndrome resulting in a natural pregnancy. J Endourol Case Rep. 2016;2:71-3.

10. Mehra S, Ranjan R, Garga UC. Zinner syndrome-a rare developmental anomaly of the mesonephric duct diagnosed on magnetic resonance imaging. Radiol Case Rep. 2016;11:313-7.

11. Jang KD, Choi KH, Yang SC, Jang WS, Jang JY, Han WK. Laparoendoscopic single-site surgery (LESS) for excision of a seminal vesicle cyst associated with ipsilateral renal agenesis. Korean J Urol. 2011;52: 431-3.

12. Canales-Casco N, Dominguez-Amillo A, Arrabal-Polo MA, Sanchez-Tamayo FJ, Arrabal-Martin M, Cozar-Olmo JM. Hematospermia as a rare form of presentation of Zinner syndrome. Urology. 2017;99:e15-e16.

13 Coskun B, Dalkiliç A, Sönmez NC, Arisan S, Ofluoglu Y, Ergenekon E. A case of seminal vesicle cyst associated with ipsilateral renal agenesis diagnosed during an investigation of urinary incontinence. Turk J Urol. 2013;39:53-5.

14. Devaraju S, Nerli RB, Hiremath MB. A large mullerian duct cyst presenting as an abdominal mass with ipsilateral renal agenesis: an unusual presentation. Nephrourol Mon. 2012;4:640-3.

15. Kao CC, Wu CJ, Sun GH, Yu DS, Chen HI, Chang SY, et al. Congenital seminal vesicle cyst associated with ipsilateral renal agenesis mimicking 
bladder outlet obstruction: a case report and review of the literature. Kaohsiung J Med Sci. 2010;26:30-4.

16. Pavan N, Bucci S, Mazzon G, Bertolotto M, Trombetta C, Liguori G. It's not always varicocele: a strange case of Zinner syndrome. Can Urol Assoc J. 2015;9:E535-8.

17. Gorrea M, Lorente R, Roel J. Seminal vesicle cyst associated with ipsilateral renal agenesis and papillary carcinoma of the bladder. Eur Radiol. 2001:11:2500-3

18. Lee BH, Seo JW, Han YH, Kim YH, Cha SJ. Primary mucinous adenocarcinoma of a seminal vesicle cyst associated with ectopic ureter and ipsilateral renal agenesis: a case report. Korean J Radiol. 2007:8:258-61.

19. Sato Y, Kataoka M, Hata J, Akaihata H, Ogawa S, Kojima Y. Renal-type clear cell carcinoma occurring in the prostate with Zinner syndrome. Urol Case Rep. 2016:5:9-12

20. Kim Y, Baek HW, Choi E, Moon KC. Squamous cell carcinoma of the seminal vesicle from zinner syndrome: a case report and review of literature. J Pathol Transl Med. 2015;49:85-8.
21. Kanavaki A, Vidal I, Merlini L, Hanquinet S. Congenital seminal vesicle cyst and ipsilateral renal agenesis (Zinner syndrome): a rare association and its evolution from early childhood to adolescence. Eur J Pediatr Surg Rep. 2015;3:98-102.

22. Aswani $\mathrm{Y}$, Varma R, Choudhary $\mathrm{P}$, Gupta RB. Wolffian origin of vagina unfolds the embryopathogenesis of OHVIRA (obstructed hemivagina and ipsilateral renal anomaly) syndrome and places OHVIRA as a female counterpart of Zinner syndrome in males. Pol J Radiol. 2016:81:549-56.

23. López García JA, Azparren Echevarría J, Garmendia G, Madina J, Garmedia C, Hernández I, et al. Seminal vesicle cyst with renal agenesis. Arch Esp Urol. 1998;51:419-26.

24. Fernández FJ, Cardoso JV, Rubio RM, Gil MJ, Martínez FC, Navarrete RV. Quiste gigante de vesícula seminal asociado con agenesia renal ipsilateral. Acta Urol Español. 2002;26:218-23.

25. Cascini V, Di Renzo D, Guerriero V, Lauriti G, Lelli Chiesa P. Zinner syndrome in pediatric age: issues in the diagnosis and treatment of a rare malformation complex. Front Pediatr. 2019;7:129. 